\title{
Index-Tunable Structured-Light Beams from a Laser with an Intracavity Astigmatic Mode Converter
}

\author{
Jing Pan $^{1,2}$, Yijie Shen ${ }^{3, *}$, Zhensong Wan ${ }^{1,2}$, Xing Fu ${ }^{1,2}$, Hengkang Zhang ${ }^{1,2}$, and Qiang $\operatorname{Liu}^{1,2}$ * \\ ${ }^{1}$ Key Laboratory of Photonic Control Technology (Tsinghua University), Ministry of Education, Beijing 100084, China \\ ${ }^{2}$ State Key Laboratory of Precision Measurement Technology and Instruments, \\ Department of Precision Instrument, Tsinghua University, Beijing 100084, China \\ ${ }^{3}$ Optoelectronics Research Centre, University of Southampton, Southampton SO17 1BJ, United Kingdom and \\ (Received 5 May 2020; revised 17 July 2020; accepted 8 September 2020; published 26 October 2020)
}

\begin{abstract}
Structured light lasers are highly topical due to not only its ability to tailor customized distribution of intensity, phase, orbital angular momentum (OAM), and other optical properties, but also the compact and simple at-the-source generation scheme. Here we propose a form of structured light laser, intracavity mode convertor laser, with the ability to generate two-dimensional (2D) tunable high-order Hermite-Gaussian (HG) modes with a large indices range (up to 15). The mode convertor constituted by two cylindrical lenses was always used for external OAM conversion, but here we show that it can be inserted into a laser cavity as an intracavity element for symmetry breaking control, which we also demonstrate with a complete matrix optics theory. The generated 2D HG modes can also be converted into Laguerre-Gaussian (LG) vortex beams with both tunable OAM and radial momentum. Moreover, we also show the ability to directly generate vortex beam from our laser cavity. Our approach meets the urgent necessity of practical laser device for more versatile light, providing new parametric space for structure control and fostering extended applications.
\end{abstract}

\section{INTRODUCTION}

In recent years, structured light, with the ability to arbitrarily tailor light in various optical properties such as intensity, phase, polarization, and orbital angular momentum (OAM), has risen in prominence 1 1. In particular it has been the emergence of vortex beams with tunable OAM [2, with the widespread applications in particle manipulation [3, 4, high-security encryption [5], quantum entanglement [6], communications [79, nanophotonics mode excitation [10 13, and nonlinear optics 14 16. Although a myriad of tools exist for structuring vortex beams external to the source [17-19, it is increasingly topical to directly create them from a laser cavity, namely structured light lasers [20, which benefits both the improved purity of beam structures and the compactness of devices. Previous works have tried to insert special intracavity elements in the forms of spatial light modulator (SLM) 21, Q-plate 22, J-plate 23], and other tailored metasurfaces [24, 25] into a laser cavity for generating tunable OAM beams. However, as for SLM, it further requires complicated digital control device and output power is limited by its low damage threshold, also SLM is highly expensive. There also are bottlenecks for other insert elements: on one hand, the intracavity elements with artificial nano-structures are highly expensive to produce, on the other hand, the generated spatial modes cannot realize two-dimensional (2D) structured tunability, i.e. the mode indices both tunable at $2 \mathrm{D}$ transverse directions. Note that the $2 \mathrm{D}$ tunable mode would have great potential to extend applications

\footnotetext{
* Corresponding author: y.shen@soton.ac.uk (Y.S.) \& qiangliu@tsinghua.edu.cn (Q.L.)
}

of structured light. For example of an Laguerre-Gaussian (LG) mode $\mathrm{LG}_{p, \ell}$, besides the well-known azimuthal in$\operatorname{dex} \ell$ related to OAM, another independent index, radial index $p$, related to the radial momentum of light, is also recently applied in quantum entanglement [26, encoding information [27, and mode sorting [28]. Therefore, the vortex mode $\mathrm{LG}_{p, \ell}$ with $2 \mathrm{D}$ tunable mode indices $(p, \ell)$ reveals the full control of azimuthal and radial momenta of light, which can largely extend optical manipulation and related applications of structured light.

To generate a vortex beam, a classic and simple way is using cylindrical lens pair as astigmatic mode converter (AMC) to transform Hermite-Gaussian (HG) mode $\mathrm{HG}_{m, n}$ into $\mathrm{LG}_{p, \ell}$ beam with index relationship of $p=\min (n, m)$ and $\ell=n-m$ 29 31]. With the AMC, the aim to generate a $\mathrm{LG}_{p, \ell}$ is equivalent to generate a $\mathrm{HG}_{m, n}$, while the required $\mathrm{HG}$ modes can be generated by using off-axis pumping in solid-state laser as an effective method 32 34. However in this way, only HG modes with one-dimension tunable order can be generated directly from cavity, because symmetry in only one dimension could be broken along the off-axis displacement of pumping, in other words, leading to the converted LG beams with tunable OAM but untunable radial index $(p=0)$ 31, 34. By insert 2D artificial mask in cavity, a 2D high-order mode can be generated [35, 36, but the fixed loss distribution prevents the mode indices from being freely tunable. In order to induce the tunable $2 \mathrm{D}$ symmetry breaking in cavity, large-aperture pumping can be exploited for tunable 2D high-order mode [37-40], but the two indices in the two dimensions are coupled to each other, which could not realize independent $2 \mathrm{D}$ tunability. In short, it still urgently requires an effective and economic scheme to generate high-order modes with freely tunable 2D indices from a structured light laser.

Here we propose a structured light laser which can con- 
trollably generate $\mathrm{HG}$ modes with $2 \mathrm{D}$-tunable indices directly from cavity and $2 \mathrm{D}$ tunable vortex beams after mode conversion, and also has ability to directly generate OAM beams. The approach is to insert an AMC, playing as an intracavity element, into a laser for tuning the $2 \mathrm{D}$ symmetry breaking in cavity by controlling offaxis displacements of intracavity elements and pumping in two dimensions independently. We also propose a complete theoretical model based on matrix optics to demonstrate the 2D mode control effect, showing great agreement with the experimental results. This work offers a unique insight into the formation of tunable structured modes in laser cavities with compact, simple, and costsaving setup scheme, which has great potential to extend related applications such as optical tweezer, imaging, and communication.

\section{EXPERIMENTAL SETUP DESIGN}

The AMC was widely utilized as an external modulator to do the conversion between HG and LG modes. But here in our structured light laser design, we applied the AMC as an intracavity element, which can impart a controlled 2D cavity symmetry breaking including different beam characteristics, gain and loss in two dimensions, due to different curvature radii of intracavity AMC and off-axis control in two dimensions. Symmetry was broken in $2 \mathrm{D}$ by elaborately putting cylindrical lenses in an off-axis-pumped laser cavity. Off-axis of cylindrical lens and pumping beam provided detuning to the cavity in two orthogonal directions, leading to dimension expending of generated HG modes. The output HG modes had tunable $2 \mathrm{D}$ indices controlled by the displacements of pump and cylindrical lenses, respectively. With AMC as the external converter of vortex beams, modes carrying OAMs with continually and independently tunable azimuthal and radial indices $(p, \ell)$ were obtained.

The experimental setup is shown in Fig. 1. The pumping source was a $976 \mathrm{~nm}$ fiber-coupled laser diode, and the gain medium was an a-cut Yb:CALGO. A concave dichroic mirror and the output coupler formed the cavity. Two cylindrical lenses $(F=25 \mathrm{~mm}$ both) inclined to $45^{\circ}$ from $y^{\prime}$-axis with $z$-axis as rotation axis were set into cavity as the control elements to stimulate a new tuning dimension of modes. Cylindrical lenses were used for controlling the direction of $\mathrm{HG}$ modes along the cylindrical lenses' s generatrix, and also for changing HG mode' s order of one dimension. Generated HG modes were converted into OAM beams through a lenses group outside the cavity. The cylindrical lens $(F=25 \mathrm{~mm})$ placed vertically was used for astigmatic conversion. We established an interference system to check the topological charges of OAM beams. Reference light was led off by a beamsplitter (BS) and expanded by a lens. OAM beams and the reference light converged through another BS to form the interference pattern, showing the topological charges of OAM beams.
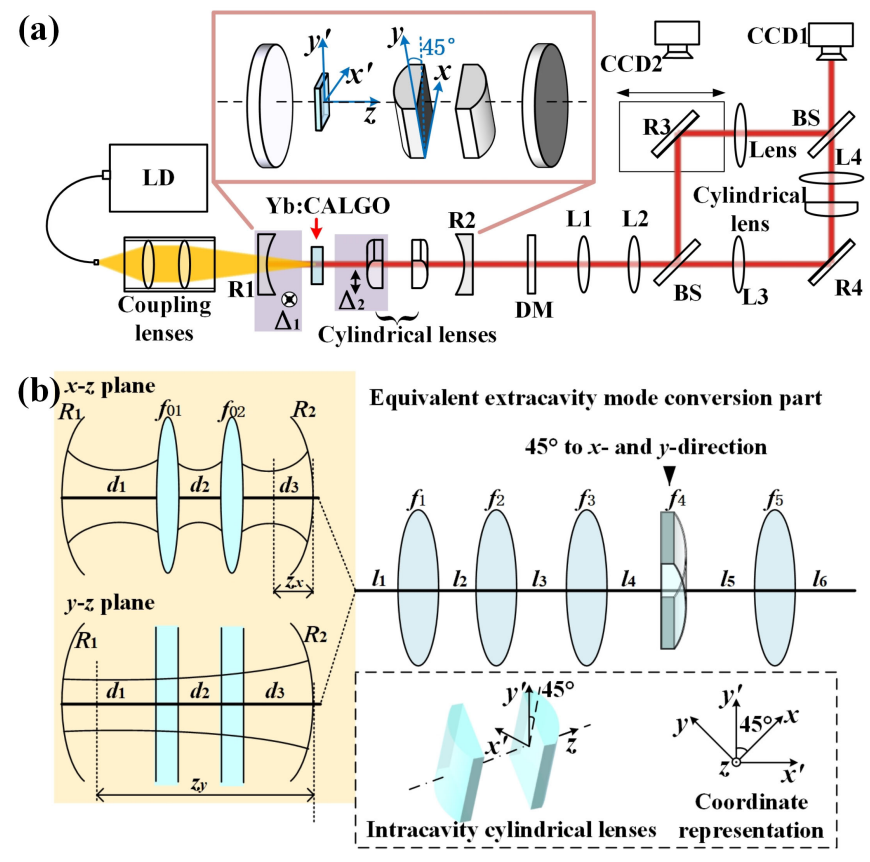

FIG. 1. The setup (a) in experiment with the insert showing the details of intracavity AMC structure, orientation of cylindrical lenses, definition of $(x, y, z)$ and $\left(x^{\prime}, y^{\prime}, z\right)$ coordinates (with the angle difference $45^{\circ}$ between $\left(x^{\prime}, y^{\prime}\right)$ and $(x, y)$ coordinates, $x^{\prime}$-, $y^{\prime}$-axis along directions of two edges of $\mathrm{Yb}$ :CALGO section respectively, $x$-axis $/ y$-axis perpendicular to/along generatrices of intracavity cylindrical lenses) and off-axis displacements of concave dichroic mirror R1 along $y^{\prime}$-axis $\left(\Delta_{1}\right)$ and the first intracavity cylindrical lens along $x^{\prime}$-axis $\left(\Delta_{2}\right)$, including LD: laser diode (Han's TCS, highest power $110 \mathrm{~W}$, with $105 \mu \mathrm{m}$ fiber core and 0.22 NA numerical aperture); coupling lenses: $976 \mathrm{~nm}$ antireflective (AR) coated lenses [focal lengths $(F): 30 \mathrm{~mm}$ and $60 \mathrm{~mm}$ ]; Yb:CALGO $\left(4 \times 4 \times 2 \mathrm{~mm}^{3}, 5\right.$ at. $\%$, AR at $\left.940-1080 \mathrm{~nm}\right) ; \mathrm{R} 1:$ concave dichroic mirror [AR at $979 \mathrm{~nm}$ and high-reflective (HR) at 1040-1080 nm, radius of curvature: $1200 \mathrm{~mm}$ ]; R2: output coupler (transmittance was $1 \%$ at $1030-1080 \mathrm{~nm}$, radius of curvature: $300 \mathrm{~mm}$ ); DM: dichroic mirror (reflectance is $99 \%$ at $979 \mathrm{~nm}$ and transmittance is $90 \%$ at $1050-1080 \mathrm{~nm}$, filter off pumping light); lenses group: lenses L1 $(F=25 \mathrm{~mm})$, L2 $(F=30 \mathrm{~mm})$, and L3 $(F=100 \mathrm{~mm}), \mathrm{L} 4(F=100 \mathrm{~mm})$; R3, R4: mirrors; BS: beamsplitter (transmittance and reflectance both are $50 \%$ at $1064 \mathrm{~nm})$; CCD: charge coupled device (Spiricon, M2-200s), with (b) equivalent light path including parameters in ABCD matrix theory and the insert of coordinate representation.

\section{THEORIES OF SYMMETRY-BREAKING MODES WITH INTRACAVITY AMC}

The intracavity AMC breaks symmetry of the cavity, because the curvature radii of cylindrical lenses are different in two perpendicular directions ( $x$-direction perpendicular to and $y$-direction along generatrices of intracavity cylindrical lenses). Different curvature radii have different effects on beam characteristics in two dimensions, such as radius of beam waist and Rayleigh length. 
Then corresponding gain and loss of the same order related with beam characteristics in two dimensions are different. The loss led in by $\mathrm{R} 1$ off-axis along $y^{\prime}$-axis has large effect to the order along $x$-axis, and the loss led in by intracavity cylindrical lens off-axis along $x^{\prime}$ axis has large effect to the order along $y$-axis. Moreover, different curvature radii of intracavity AMC also lead in special Gouy phase difference to two directions. Based on the above two points, corresponding 2D HG modes are selected, and after conversion, homologous 2D OAM modes are obtained. Thus orders are both tunable in two dimensions independently, and directions of two dimensions are depended on the set intracavity AMC.

Besides mode indices tunable in two dimensions, intracavity AMC also incidentally introduces astigmatism into cavity, which makes practical modes different from standard modes in ellipticity of transverse intensity profile. Thus, we use "OAM mode" distinguishing practical mode from standard LG mode. ABCD matrix optics theory is a suitable method to analyse astigmatism in practical modes. Practical beam characteristics can be obtained based on the matrix theory. With the practical beam characteristics in mode functions, astigmatic modes can be obtained in theory.

For the intracavity part, due to different beam characteristics in two dimensions caused by intracavity AMC, we combine ABCD matrix and parameter $q$ with HG function in different two dimensions to analyse output $\mathrm{HG}_{m, n}$ modes. From the light path of intracavity part shown in experimental setup, intracavity AMC is the only symmetry breaking element of the intracavity part and axes of two cylindrical lenses in cavity are parallel to each other. Thus two dimensions in the intracavity part are independent and ABCD matrices in cavity of two dimensions can be treated separately 41,43 . One-round trip $\mathrm{ABCD}$ matrices of the cavity perpendicular to $(\mathbf{X})$ and parallel with $(\mathbf{Y})$ the generatrices of cylindrical lenses are given as:

$$
\begin{array}{r}
\mathbf{X}=\mathbf{M}\left(d_{3}\right) \mathbf{N}\left(-\frac{1}{f_{02}}\right) \mathbf{M}\left(d_{2}\right) \mathbf{N}\left(-\frac{1}{f_{01}}\right) \\
\cdot \mathbf{M}\left(d_{1}\right) \mathbf{N}\left(-\frac{2}{R_{1}}\right) \mathbf{M}\left(d_{1}\right) \mathbf{N}\left(-\frac{1}{f_{01}}\right) \\
\cdot \mathbf{M}\left(d_{2}\right) \mathbf{N}\left(-\frac{1}{f_{02}}\right) \mathbf{M}\left(d_{3}\right) \mathbf{N}\left(-\frac{2}{R_{2}}\right) \\
\mathbf{Y}=\mathbf{M}\left(d_{3}\right) \mathbf{M}\left(d_{2}\right) \mathbf{M}\left(d_{1}\right) \mathbf{N}\left(-\frac{2}{R_{1}}\right) \\
\cdot \mathbf{M}\left(d_{1}\right) \mathbf{M}\left(d_{2}\right) \mathbf{M}\left(d_{3}\right) \mathbf{N}\left(-\frac{2}{R_{2}}\right)
\end{array}
$$

where $\mathbf{M}(x)=\left[\begin{array}{ll}1 & x \\ 0 & 1\end{array}\right], \quad \mathbf{N}(x)=\left[\begin{array}{ll}1 & 0 \\ x & 1\end{array}\right], \quad \mathbf{X}=\left[\begin{array}{ll}A_{x} & B_{x} \\ C_{x} & D_{x}\end{array}\right]$, $\mathbf{Y}=\left[\begin{array}{ll}A_{y} & B_{y} \\ C_{y} & D_{y}\end{array}\right] . \quad d_{1}$ to $d_{3}$ are distances between $\mathrm{R} 1$ and the first cylindrical lens, two cylindrical lenses, the second cylindrical lens and R2. $R_{1}$ and $R_{2}$ are curvature radii of $\mathrm{R} 1$ and $\mathrm{R} 2 . f_{01}$ and $f_{02}$ are focal lengths of two cylindrical lenses, as Fig. 11(b).

Based on the relation between $q_{\xi}$ (parameter $q$ of beams in $\xi$-direction at position $\left.Z_{\xi}, \xi=x, y\right)$ and $\mathrm{ABCD}$ matrix in cavity $q_{\xi 1}=\frac{A_{\xi} q_{\xi}+B_{\xi}}{C_{\xi} q_{\xi}+D_{\xi}}$ (parameter $q_{\xi 1}$ is parameter $q$ of beams in $\xi$-direction at a new position $Z_{\xi 1}$ after transmission from the old position $Z_{\xi}$ ), and beam selfreproduction $q_{\xi 1}=q_{\xi}$, parameter $1 / q_{\xi}$ can be expressed by $\mathrm{ABCD}$ matrix as:

$$
\frac{1}{q_{\xi}}=\frac{D_{\xi}-A_{\xi}}{2 B_{\xi}}-i \frac{1}{\left|B_{\xi}\right|} \sqrt{1-\left(\frac{A_{\xi}+D_{\xi}}{2}\right)^{2}}
$$

The imaginary part of complex parameter $q_{\xi}$ is $q_{0 \xi}$, and the real part of complex parameter $q_{\xi}$ is the distance away from beam waist $z_{\xi}$, shown as:

$$
\begin{gathered}
q_{0 \xi}=\frac{i \frac{1}{\mid B_{\xi}} \sqrt{1-\left(\frac{A_{\xi}+D_{\xi}}{2}\right)^{2}}}{\left(\frac{D_{\xi}-A_{\xi}}{2 B_{\xi}}\right)^{2}+\frac{1}{\left|B_{\xi}\right|^{2}}\left[1-\left(\frac{A_{\xi}+D_{\xi}}{2}\right)^{2}\right]} \\
z_{\xi}=\frac{\frac{D_{\xi}-A_{\xi}}{2 B_{\xi}}}{\left(\frac{D_{\xi}-A_{\xi}}{2 B_{\xi}}\right)^{2}+\frac{1}{\left|B_{\xi}\right|^{2}}\left[1-\left(\frac{A_{\xi}+D_{\xi}}{2}\right)^{2}\right]}
\end{gathered}
$$

Radius of beam waist $\omega_{0 \xi}$ with the order $\nu$ is $\sqrt{2 \nu+1}$ times that of fundamental mode. Based on the relation between $q_{0 \xi}$ and radius of beam waist $\omega_{0 \xi}$ and Rayleigh length $z_{R_{\xi}}$, the radius of beam waist is $\omega_{0 \xi}=$ $\sqrt{-i(2 \nu+1) q_{0 \xi} \lambda / \pi}$, and the Rayleigh length $z_{R_{\xi}}$ is $z_{R_{\xi}}=\pi \omega_{0 \xi}{ }^{2} / \lambda$. Practical beam characteristics $\omega_{0 \xi}, z_{R_{\xi}}$ can be obtained by substituting $q_{0 \xi}$ of Eq. (4).

HG mode solved from Helmholtz equation with the order $m$ along $x$-axis, the order $n$ along $y$-axis is given by:

$$
\Psi_{m, n}^{(\mathrm{HG})}(x, y)=\left(2^{m+n-1} \pi m ! n !\right)^{-1 / 2} \psi_{m}(x) \psi_{n}(y)
$$

HG function in one dimension could yield $(\xi=x, \nu=m$; $\xi=y, \nu=n)$

$$
\begin{aligned}
\psi_{\nu}(\xi)=\frac{e^{-\frac{\xi^{2}}{\omega_{\xi}^{2}\left(z_{\xi}\right)}}}{\sqrt{\omega_{\xi}\left(z_{\xi}\right)}} H_{\nu}\left[\frac{\sqrt{2} \xi}{\omega_{\xi}\left(z_{\xi}\right)}\right] e^{i k \frac{\xi^{2}}{2 R_{\xi}\left(z_{\xi}\right)}} \\
\times e^{-i\left(\nu+\frac{1}{2}\right) \tan ^{-1}\left(\frac{z_{\xi}}{z_{R_{\xi}}}\right)}
\end{aligned}
$$

where radius of beam $\omega_{\xi}\left(z_{\xi}\right)=\omega_{0 \xi} \sqrt{1+\left(z_{\xi} / z_{R_{\xi}}\right)^{2}}$, and radius of equiphase surface $R_{\xi}\left(z_{\xi}\right)=z_{\xi}\left(1+z_{R_{\xi}}^{2} / z_{\xi}^{2}\right)$ at $z_{\xi}$. By substituting beam characteristics $\omega_{0 \xi}, z_{R_{\xi}}, z_{\xi}$ obtained above to Eq. (5), actually acquired HG modes can be simulated.

Comparing with Fig. 2(a) the standard HG mode, by assigning $m=3, n=2$ in Eq. (5), HG modes directly from cavity can be obtained in simulation as shown in 

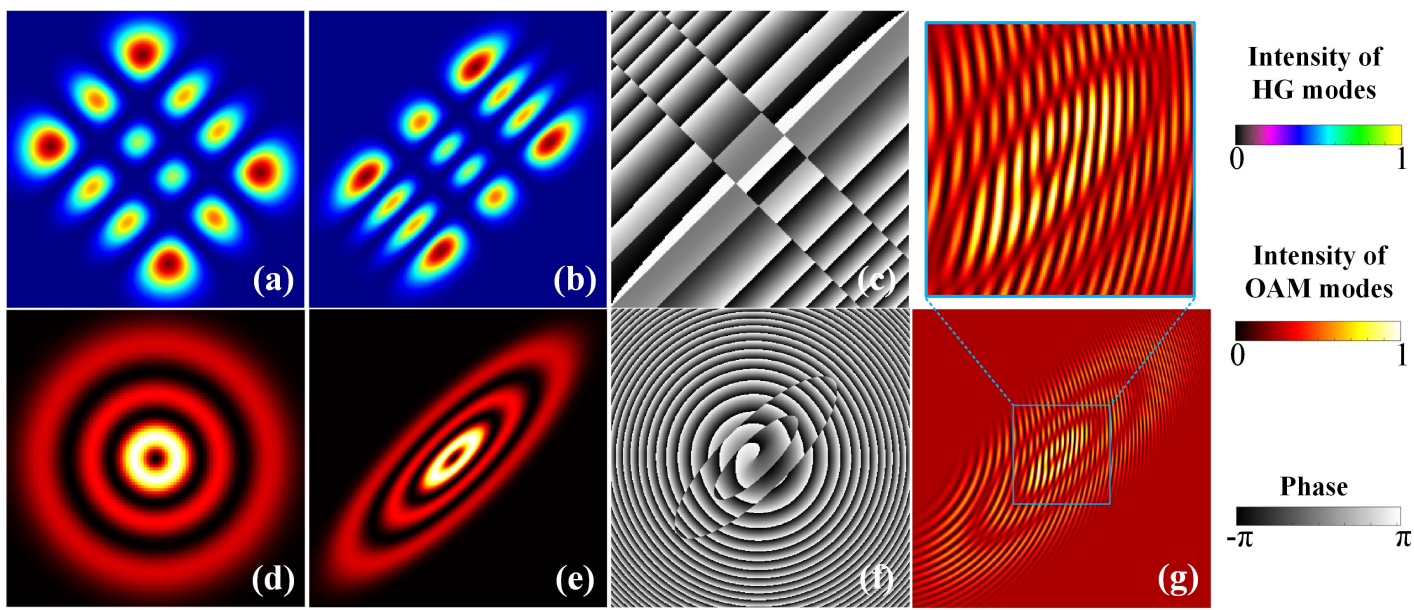

FIG. 2. Simulated results of the structured laser beams: (a) Standard $\mathrm{HG}_{3,2}$ mode; (b) $\mathrm{HG}_{3,2}$ mode directly from intracavity AMC laser and (c) its phase. (d) Standard $\mathrm{LG}_{2,-1}$ mode. (e) OAM $\mathrm{OA}_{2,-1}$ mode converted after intracavity AMC laser and (f) its phase. (g) The interference pattern of $\mathrm{OAM}_{2,-1}$ mode and reference beam, the insert shows the zoom-in figure of the coherent fringes.

Fig. 2(b), with $\mathrm{HG}_{3,2}$ as an example. We could find that besides the expand of mode dimension, intracavity AMC also leads astigmatism into HG modes.

As for the extracavity mode conversion part, due to the external cylindrical lens set at $45^{\circ}$ (extracavity cylindrical lens rotated angle $\theta$ ) to $\mathrm{HG}$ modes, it induces Gouy phase difference into two dimensions, converting HG modes into OAM modes. Different from ABCD matrix analysed independently in cavity in two dimensions, extracavity ABCD matrix of two dimensions could not be treated independently, due to cylindrical lens outside cavity are not parallel with cylindrical lenses in cavity. Therefore, an expansion matrix related to extracavity cylindrical lens' $\mathrm{s}$ angle of rotation is used to describe beam transmission outside cavity, shown as:

$$
\begin{aligned}
\mathbf{T}= & \mathbf{P}\left(l_{6}, l_{6}\right) \mathbf{Q}\left(-\frac{1}{f_{5}}\right) \mathbf{P}\left(l_{5}, l_{5}\right) \mathbf{R}\left(\frac{1}{f_{4}}\right) \\
& \cdot \mathbf{P}\left(l_{4}, l_{4}\right) \mathbf{Q}\left(-\frac{1}{f_{3}}\right) \mathbf{P}\left(l_{3}, l_{3}\right) \mathbf{Q}\left(-\frac{1}{f_{2}}\right) \\
& \cdot \mathbf{P}\left(l_{2}, l_{2}\right) \mathbf{Q}\left(-\frac{1}{f_{1}}\right) \mathbf{P}\left(l_{1}+z_{x}, l_{1}+z_{y}\right)
\end{aligned}
$$

where $\quad \mathbf{P}(x, y)=\left[\begin{array}{llll}1 & 0 & x & 0 \\ 0 & 1 & 0 & y \\ 0 & 0 & 1 & 0 \\ 0 & 0 & 0 & 1\end{array}\right], \quad \mathbf{Q}(x)=\left[\begin{array}{llll}1 & 0 & 0 & 0 \\ 0 & 1 & 0 & 0 \\ x & 0 & 1 & 0 \\ 0 & x & 0 & 1\end{array}\right]$, $\mathbf{R}(x)=\left[\begin{array}{cccc}1 & 0 & 0 & 0 \\ 0 & 1 & 0 & 0 \\ -x \cos ^{2} \alpha & \frac{x \sin 2 \alpha}{2} & 1 & 0 \\ \frac{x \sin 2 \alpha}{2} & -x \sin ^{2} \alpha & 0 & 1\end{array}\right] \quad$ 44 $\quad$ related to rotated cylindrical lens with rotated angle $\alpha=45^{\circ} \cdot l_{1}$ to $l_{6}$ are distances between R2, L1, L2, L3, cylindrical lens, L4 and CCD1 in turn. $f_{1}$ to $f_{3}$, and $f_{5}$ are focal lengths of L1 to L4, and $f_{4}$ is focal length of cylindrical lens. $z_{x}$ and $z_{y}$ are distances between R2 and corresponding beam waist of beam directly from cavity based on Eq. (4), as Fig. 1(b).

With extracavity ABCD expansion matrix in Eq. (7) and parameter $q_{0 \xi}$ of modes directly from cavity in Eq. (4), the relation between parameters $q_{\xi}$ of beams after conversion and extracavity ABCD expansion matrix are shown as

$$
\begin{aligned}
q_{x} & =\frac{T_{11} q_{0 x}+T_{13}}{T_{31} q_{0 x}+T_{33}} \\
q_{y} & =\frac{T_{22} q_{0 y}+T_{24}}{T_{42} q_{0 y}+T_{44}}
\end{aligned}
$$

where $\mathbf{T}(i, j)=T_{i j}$. Beam characteristics are obtained with $q_{\xi}$ in Eq. (8). The radius of beam waist is $\omega_{0 \xi}=$ $\sqrt{-i q_{0 \xi} \lambda / \pi}$, where $q_{0 \xi}$ is the imaginary part of $q_{\xi}$ in Eq. 8), and the Rayleigh length $z_{R_{\xi}}$ is $z_{R_{\xi}}=\pi \omega_{0 \xi}{ }^{2} / \lambda$. The real part of $q_{\xi}$ in Eq. (8) is the distance $z_{\xi}$ from corresponding beam waist.

Modes carrying OAMs after astigmatic conversion outside the cavity are written as [45, 46]:

$$
\Psi^{(\mathrm{OAM})}\left(x^{\prime}, y^{\prime}\right)=\sum_{s=0}^{N} d_{s-\frac{N}{2}, n-\frac{N}{2}}^{\frac{N}{2}}(\theta) \Psi_{s, N-s}^{(\mathrm{HG})}(x, y) e^{-i s \beta}
$$

where the elements of Wigner $d$-matrix are given by:

$$
\begin{aligned}
& d_{s-\frac{N}{2}, n-\frac{N}{2}}^{\frac{N}{2}}(\theta)=\sqrt{s !} \sqrt{(N-s) ! \sqrt{n !} \sqrt{(N-n) !}} \\
& \times \sum_{v=\max [0, s-n]}^{\min [N-n, s]} \frac{(-1)^{v}[\cos (\theta)]^{m+s-2 v}[\sin (\theta)]^{n-s+2 v}}{v !(N-n-v) !(s-v) !(n-s+v) !}
\end{aligned}
$$

$N=n+m, x^{\prime}=x \cos \theta+y \sin \theta, y^{\prime}=x \sin (-\theta)+$ $y \cos \theta$. Gouy phase difference of two dimensions $\beta=\frac{\pi}{2}+$ 
$\tan ^{-1}\left(\frac{z_{x}}{z_{R_{x}}}\right)-\tan ^{-1}\left(\frac{z_{y}}{z_{R_{y}}}\right)$. By substituting practical beam characteristics $\omega_{0 \xi}, z_{R_{\xi}}, z_{\xi}$ based on Eq. (8) to Eq. (9), OAM modes in the simulation could be obtained.

Comparing with Fig. 2(d) standard LG mode, by assigning $m=3, n=2$ in Eq. (9), OAM OA,-1 $_{2}$ converted from $\mathrm{HG}_{3,2}$ shown in Fig. 2(e) was taken as an example.

The simulation deepens the understanding of astigmatic OAM modes, which are different from the standard LG modes in ellipticity of their transverse intensity profile, expanding the mode patterns as well as applications.

\section{EXPERIMENTAL RESULTS AND DISCUSSION}

With experimental setup shown in Fig. 1, when all cavity elements were coaxial, fundamental mode was obtained at the pumping threshold of $0.56 \mathrm{~W}$. With different off-axis displacements of intracavity cylindrical lens and R1, different loss and gain were brought in two dimensions, and output HG modes were selected as Fig. 3 . recorded by CCD2 with R3 moved off. The specific relation between obtained astigmatic $\mathrm{HG}$ modes $\mathrm{HG}_{m, n}$ and different off-axis displacement of R1 along $y^{\prime}$-axis $\Delta_{1}$, off-axis displacement of the first cylindrical lens along $x^{\prime}$-axis $\Delta_{2}$ and the pumping power $P$ was shown as Table $\mathrm{I}$ in appendix. Modes like $\mathrm{HG}_{0, i}(i=9-12,14), \mathrm{HG}_{1, i}$ $(i=1,3), \mathrm{HG}_{2,5}$, and $\mathrm{HG}_{4,4}$ were also generated. However, for the case of very high-order modes (generally one index is larger than 10 or two indices both are not 0 ), due to the small difference of loss between two adjacent modes and the limitation of experimental adjustment accuracy of instrument, these modes could not be all stably captured, and thus we just show some relatively stable results as representatives.

From Fig. 3, HG modes could be controllably generated in two dimensions directly from the laser cavity. HG mode indices $m$ and $n$ were tunable continually and independently, changing with R1 off-axis along $y^{\prime}$-axis or one of the cylindrical lenses off-axis along $x^{\prime}$-axis, respectively. The varying range of $m$ was from 0 to 4 , and in total, output HG modes were in a large tunable range (more than 20 modes). The tunable range was limited by pumping power due to the relation between gain and loss, and with the increase of pumping power, tunable range would enlarge. The output power of $\mathrm{HG}$ modes could be adjusted with pumping power, and with experimental pumping power, the output power was in milliwatt level. Moreover, shown as Fig. 3, direction of $\mathrm{HG}$ modes was limited by the setting angle of the intracavity cylindrical lenses. Two dimensions were parallel to and perpendicular to the generatrix of intracavity AMC.

Generated certain $\mathrm{HG}_{m, n}$ mode was converted into corresponding $\mathrm{OAM}_{p, \ell}$ mode after mode conversion by going through the extracavity lens group (L1, L2, L3, cylindrical lens, and L4) as shown in the setup in Fig. 1 . The radial index $p$ is the smaller one of two parameters $m$ and $n$, and the azimuthal index $\ell$ is $n$ - $m$. Homologous

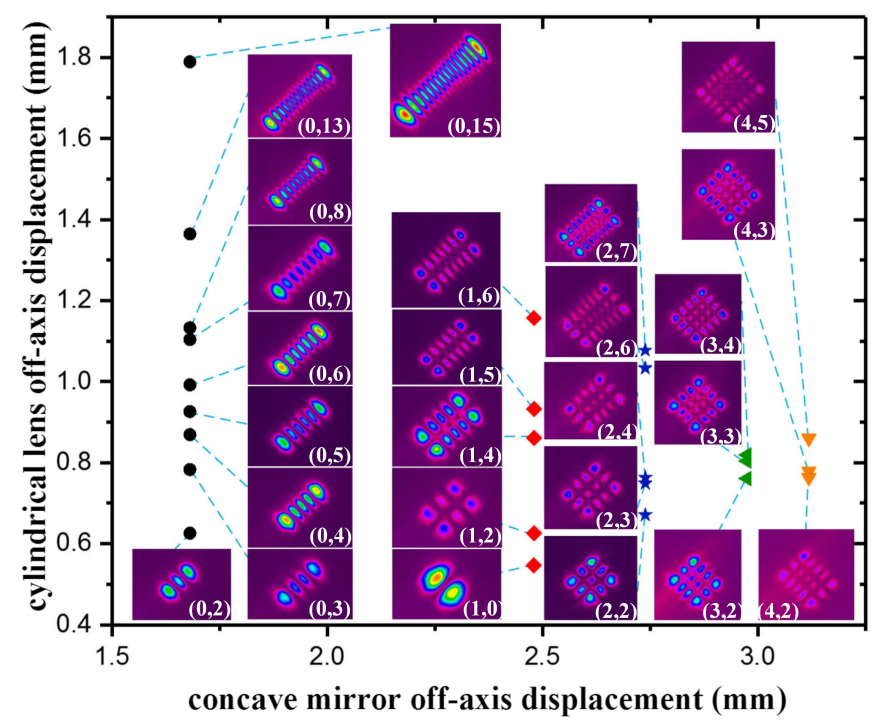

FIG. 3. The experimental results of the evolution of different generated HG modes with continually and independently tunable indices $(m, n)$ with the off-axis of the cylindrical lens and the concave mirror.

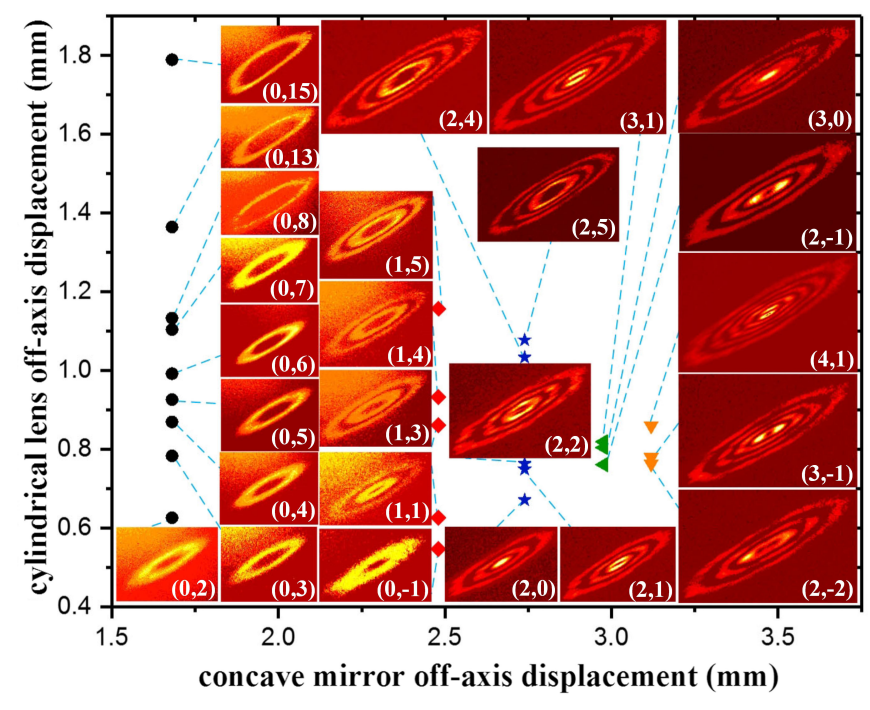

FIG. 4. The experimental results of the evolution of different OAM modes carrying continually and independently tunable radial index and azimuthal index $(p, \ell)$ with the homologous off-axis displacements of the cylindrical lens and the concave mirror of HG modes.

OAM modes were recorded by CCD1, whose indices were stated specifically in Table Tin appendix. In Fig. 4. OAM beams with continually and independently tunable radial and azimuthal indices $(p, \ell)$ were shown clearly. Limited by the generated $\mathrm{HG}$ modes, the tunable range of radial index $p$ was 0 to 4 , and azimuthal index $\ell$ could be changed from -1 to 15,0 to $5,-2$ to $5,-1$ to 1,0 to 1 respectively. OAM modes range could be changed with HG modes tunable range.

Output power of OAM modes was also in milliwatt 


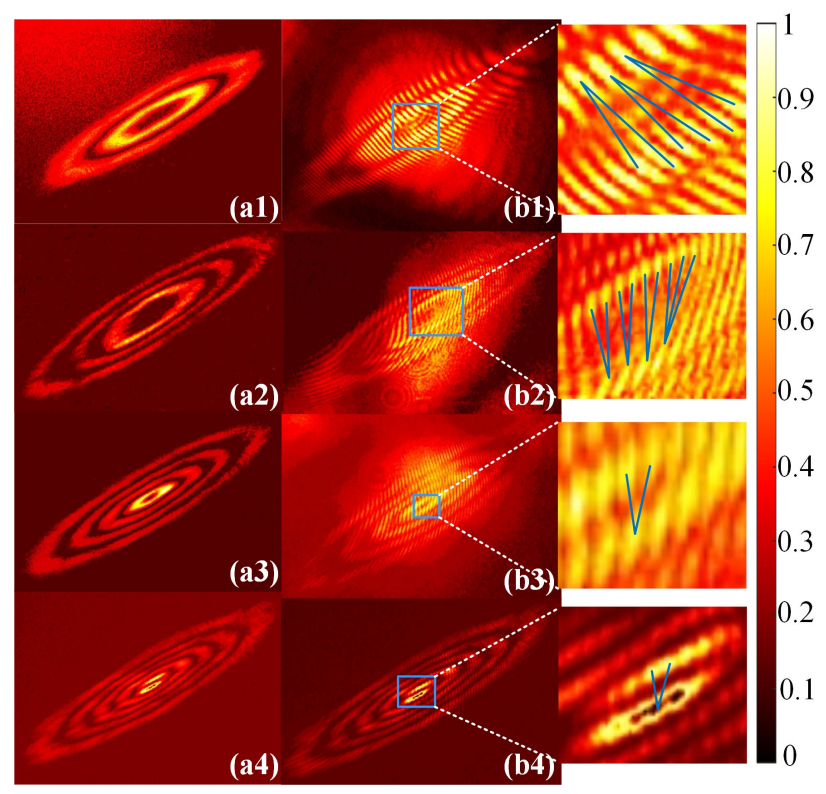

FIG. 5. The experimental results of (a1-a4) $\mathrm{OAM}_{1,3}, \mathrm{OAM}_{2,5}$, $\mathrm{OAM}_{3,1}, \mathrm{OAM}_{4,1}$ and (b1-b4) corresponding interference patterns. The inserts show the zoom-in figures of the coherent fringes in figures (b1-b4).

level with a high conversion efficiency from HG modes. Moreover, different from standard LG modes, output OAM modes were astigmatic obviously in Fig. 4 among which $\mathrm{OAM}_{2,-1}$ was consistent with its simulational result in Fig. 2(e). Ellipticity in transverse intensity profile of OAM modes in Fig. 4 is in the range of 0.19 to 0.28 .

Angular momentum of OAM modes could be verified by interference patterns with reference beam. In our experiment, reference light was the flat part of beam which was led off by BS and expanded by lens. Interference patterns were recorded by CCD1 as illustrated in Fig. 5 . $\mathrm{OAM}_{1,3}, \mathrm{OAM}_{2,5}, \mathrm{OAM}_{3,1}, \mathrm{OAM}_{4,1}$ in Fig. 5(a1-a4) were taken as examples for their different radial index $p$, with their interference patterns in Fig. 5(b1-b4). The number of forks showed the topological charges three, five, one, one in each circle, respectively, corresponding to azimuthal index $\ell$.

As an additional function of this cavity structure with the pair of cylindrical lenses set vertically in the cavity, OAM beams carrying $\pm 1 \hbar$ could be generated directly from the cavity. Based on the light path above, the pair of cylindrical lenses were set $35.7 \mathrm{~mm}$ apart from each other between Yb:CALGO and R2. $\Delta_{1}$ and $\Delta_{2}$ were controlled as $0.847 \mathrm{~mm}$ and $0.068 \mathrm{~mm}$ respectively at the pumping power $4.01 \mathrm{~W}$. Without the cylindrical lens outside the cavity, mode was recorded by CCD1 shown in Fig. 6(a). Its interference pattern was recorded by CCD1 shown in Fig. 6(b). The number of fork showed generated mode carrying $\pm 1 \hbar$ OAM. Simulational result in Fig. 6(c) was obtained from Eq. (9) as $-\frac{1}{f_{4}}=0$ and $m=0, n=1$. For the case of generating 2D HG modes, the Gouy phase difference of beam between $x$-, $y$-directions was so small
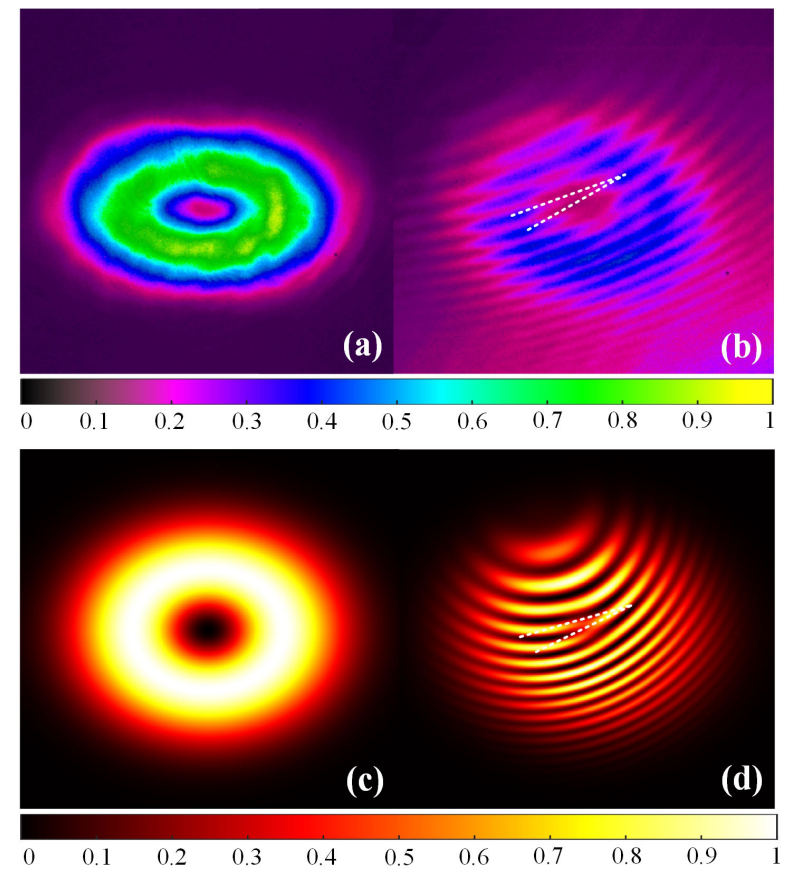

FIG. 6. The experimental results of (a) $\pm 1 \hbar$ OAM mode generated directly from the cavity and (b) the corresponding interference pattern. The simulated verifications of (c) the $\pm 1 \hbar$ OAM mode and (d) the interference pattern.

that only HG modes could be generated. While, for the case of generating OAM mode, we elaborately adjusted the positions of the intracavity cylindrical lenses along $z$-axis, so that the Gouy phase difference between two orthogonal directions was nearly $\pi / 2$, which allowed the transformation from HG mode into OAM mode in cavity and the direct generation of OAM mode.

\section{CONCLUSION}

In conclusion, we used AMC as an intracavity element to construct a form of structured light laser to generate $2 \mathrm{D}$ tunable high-order modes and vortex beams. In our approach, the 2D symmetry-breaking was controllable by tuning off-axis displacements of intracavity AMC and cavity mirror, which led to independent tunability of modes in 2D. A complete theoretical model combining $\mathrm{ABCD}$ matrix and modal astigmatism is proposed to simulate the structured laser, which is well verified by experimental results. The generated $\mathrm{HG}_{m, n}$ modes can be tuned continually and independently in two dimensions, with tunable indices up to 15. After external astigmatic conversion, OAM beams carrying continually and independently tunable radial and azimuthal indices $(p, \ell)$ were obtained, with tunable range of $p$ as 0 to 4 , and that of $\ell$ from -2 to 15 . It is the realization of vortex beam with both tunable orbital angular and radial momenta simultaneously. Additionally, $\pm 1 \hbar$ OAM modes could be generated directly from the cavity by proper control. This 
work has great potential in increasing structured light tunability at the source in a simple and cost-saving way, and providing deeper physical insight on understanding $2 \mathrm{D}$ practical astigmatic modes and enriching the related applications.

\section{ACKNOWLEDGMENTS}

National Natural Science Foundation of China (61875100), National Natural Science Foundation of China (61975087), National Key Research and Development Program of China (2017YFB1104500), Beijing Young Talents Support Project (2017000020124G044), and Marie S.-Curie MULTIPLY Fellowship in Europe (GA713694).

Appendix A: Table of obtained modes 
TABLE I. Pumping power and off-axis displacements for different HG modes and OAM modes

\begin{tabular}{|c|c|c|c|c|c|c|c|}
\hline \multicolumn{4}{|c|}{$\Delta_{1}=1.681 \mathrm{~mm}$ (round dots row) } & \multicolumn{4}{|c|}{$\Delta_{1}=2.739 \mathrm{~mm}$ (star dots row) } \\
\hline$\Delta_{2}(m m)$ & $\mathrm{P}(\mathrm{W})$ & $\mathrm{HG}_{m, n}$ & $\mathrm{OAM}_{p, \ell}$ & $\Delta_{2}(m m)$ & $\mathrm{P}(\mathrm{W})$ & $\mathrm{HG}_{m, n}$ & $\mathrm{OAM}_{p, \ell}$ \\
\hline 0.626 & 2.95 & $\mathrm{HG}_{0,2}$ & $\mathrm{OAM}_{0,2}$ & 0.671 & 5.49 & $\mathrm{HG}_{2,2}$ & $\mathrm{OAM}_{2,0}$ \\
\hline 0.783 & 3.37 & $\mathrm{HG}_{0,3}$ & $\mathrm{OAM}_{0,3}$ & 0.749 & 6.06 & $\mathrm{HG}_{2,3}$ & $\mathrm{OAM}_{2,1}$ \\
\hline 0.869 & 3.80 & $\mathrm{HG}_{0,4}$ & $\mathrm{OAM}_{0,4}$ & 0.763 & 6.16 & $\mathrm{HG}_{2,4}$ & $\mathrm{OAM}_{2,2}$ \\
\hline 0.926 & 4.01 & $\mathrm{HG}_{0,5}$ & $\mathrm{OAM}_{0,5}$ & 1.033 & 7.93 & $\mathrm{HG}_{2,6}$ & $\mathrm{OAM}_{2,4}$ \\
\hline 0.992 & 4.22 & $\mathrm{HG}_{0,6}$ & $\mathrm{OAM}_{0,6}$ & 1.077 & 8.69 & $\mathrm{HG}_{2,7}$ & $\mathrm{OAM}_{2,5}$ \\
\hline 1.104 & 4.43 & $\mathrm{HG}_{0,7}$ & $\mathrm{OAM}_{0,7}$ & \multicolumn{4}{|c|}{$\Delta_{1}=2.975 \mathrm{~mm}$ (regular triangle dots row) } \\
\hline 1.133 & 4.43 & $\mathrm{HG}_{0,8}$ & $\mathrm{OAM}_{0,8}$ & $\Delta_{2}(\mathrm{~mm})$ & $\mathrm{P}(\mathrm{W})$ & $\mathrm{HG}_{m, n}$ & $\mathrm{OAM}_{p, \ell}$ \\
\hline 1.364 & 5.77 & $\mathrm{HG}_{0,13}$ & $\mathrm{OAM}_{0,13}$ & 0.761 & 7.80 & $\mathrm{HG}_{3,2}$ & $\mathrm{OAM}_{2,-1}$ \\
\hline 1.789 & 7.41 & $\mathrm{HG}_{0,15}$ & $\mathrm{OAM}_{0,15}$ & 0.804 & 8.06 & $\mathrm{HG}_{3,3}$ & $\mathrm{OAM}_{3,0}$ \\
\hline \multicolumn{4}{|c|}{$\Delta_{1}=2.480 \mathrm{~mm}$ (diamond dots row) } & 0.819 & 8.99 & $\mathrm{HG}_{3,4}$ & $\mathrm{OAM}_{3,1}$ \\
\hline$\Delta_{2}(m m)$ & $\mathrm{P}(\mathrm{W})$ & $\mathrm{HG}_{m, n}$ & $\mathrm{OAM}_{p, \ell}$ & \multicolumn{4}{|c|}{$\Delta_{1}=3.119 \mathrm{~mm}$ (inverted triangle dots row) } \\
\hline 0.547 & 4.32 & $\mathrm{HG}_{1,0}$ & $\mathrm{OAM}_{0,-1}$ & $\Delta_{2}(\mathrm{~mm})$ & $\mathrm{P}(\mathrm{W})$ & $\mathrm{HG}_{m, n}$ & $\mathrm{OAM}_{p, \ell}$ \\
\hline 0.626 & 4.97 & $\mathrm{HG}_{1,2}$ & $\mathrm{OAM}_{1,1}$ & 0.763 & 8.83 & $\mathrm{HG}_{4,2}$ & $\mathrm{OAM}_{2,-2}$ \\
\hline 0.861 & 5.86 & $\mathrm{HG}_{1,4}$ & $\mathrm{OAM}_{1,3}$ & 0.779 & 8.83 & $\mathrm{HG}_{4,3}$ & $\mathrm{OAM}_{3,-1}$ \\
\hline 0.933 & 7.14 & $\mathrm{HG}_{1,5}$ & $\mathrm{OAM}_{1,4}$ & 0.860 & 10.03 & $\mathrm{HG}_{4,5}$ & $\mathrm{OAM}_{4,1}$ \\
\hline 1.157 & 8.83 & $\mathrm{HG}_{1,6}$ & $\mathrm{OAM}_{1,5}$ & & & & \\
\hline
\end{tabular}


[1] Halina Rubinsztein-Dunlop, Andrew Forbes, Michael V Berry, Mark R Dennis, David L Andrews, Masud Mansuripur, Cornelia Denz, Christina Alpmann, Peter Banzer, Thomas Bauer, et al., "Roadmap on structured light," Journal of Optics 19, 013001 (2016).

[2] Yijie Shen, Xuejiao Wang, Zhenwei Xie, Changjun Min, Xing Fu, Qiang Liu, Mali Gong, and Xiaocong Yuan, "Optical vortices 30 years on: OAM manipulation from topological charge to multiple singularities," Light: Science \& Applications 8, 1-29 (2019).

[3] Miles Padgett and Richard Bowman, "Tweezers with a twist," Nature Photonics 5, 343-348 (2011).

[4] A Lafong, WJ Hossack, J Arlt, TJ Nowakowski, and ND Read, "Time-Multiplexed Laguerre-Gaussian holographic optical tweezers for biological applications," Optics Express 14, 3065-3072 (2006).

[5] Xinyuan Fang, Haoran Ren, and Min Gu, "Orbital angular momentum holography for high-security encryption," Nature Photonics 14, 102-108 (2020).

[6] Alois Mair, Alipasha Vaziri, Gregor Weihs, and Anton Zeilinger, "Entanglement of the orbital angular momentum states of photons," Nature 412, 313 (2001).

[7] Jian Wang, Jeng-Yuan Yang, Irfan M Fazal, Nisar Ahmed, Yan Yan, Hao Huang, Yongxiong Ren, Yang Yue, Samuel Dolinar, Moshe Tur, et al., "Terabit freespace data transmission employing orbital angular momentum multiplexing," Nature Photonics 6, 488 (2012).

[8] Nenad Bozinovic, Yang Yue, Yongxiong Ren, Moshe Tur, Poul Kristensen, Hao Huang, Alan E Willner, and Siddharth Ramachandran, "Terabit-scale orbital angular momentum mode division multiplexing in fibers," Science 340, 1545-1548 (2013).

[9] Jian Wang, "Advances in communications using optical vortices," Photonics Research 4, B14-B28 (2016).

[10] RM Kerber, JM Fitzgerald, X Xiao, Sang Soon Oh, SA Maier, V Giannini, and DE Reiter, "Interaction of an Archimedean spiral structure with orbital angular momentum light," New Journal of Physics 20, 095005 (2018).

[11] Guillermo Federico Quinteiro and Pablo Ignacio Tamborenea, "Electronic transitions in disk-shaped quantum dots induced by twisted light," Physical Review B 79, 155450 (2009).

[12] Richard M Kerber, Jamie M Fitzgerald, Doris E Reiter, Sang Soon Oh, and Ortwin Hess, "Reading the orbital angular momentum of light using plasmonic nanoantennas," ACS Photonics 4, 891-896 (2017).

[13] Yuan Meng, Zhoutian Liu, Zhenwei Xie, Ride Wang, Tiancheng Qi, Futai Hu, Hyunseok Kim, Qirong Xiao, Xing Fu, Qiang $\mathrm{Wu}$, et al., "Versatile on-chip light coupling and (de) multiplexing from arbitrary polarizations to controlled waveguide modes using an integrated dielectric metasurface," Photonics Research 8, 564-576 (2020).

[14] Laura Rego, Kevin M Dorney, Nathan J Brooks, Quynh L Nguyen, Chen-Ting Liao, Julio San Román, David E Couch, Allison Liu, Emilio Pisanty, Maciej Lewenstein, et al., "Generation of extreme-ultraviolet beams with time-varying orbital angular momentum," Science 364, eaaw9486 (2019).

[15] Kevin M Dorney, Laura Rego, Nathan J Brooks, Julio San Román, Chen-Ting Liao, Jennifer L Ellis, Dmitriy
Zusin, Christian Gentry, Quynh L Nguyen, Justin M Shaw, et al., "Controlling the polarization and vortex charge of attosecond high-harmonic beams via simultaneous spin-orbit momentum conservation," Nature Photonics 13, 123-130 (2019).

[16] Can Huang, Chen Zhang, Shumin Xiao, Yuhan Wang, Yubin Fan, Yilin Liu, Nan Zhang, Geyang Qu, Hongjun Ji, Jiecai Han, et al., "Ultrafast control of vortex microlasers," Science 367, 1018-1021 (2020).

[17] Wei Ji, Chun-Hong Lee, Peng Chen, Wei Hu, Yang Ming, Lijian Zhang, Tsung-Hsien Lin, Vladimir Chigrinov, and Yan-Qing Lu, "Meta-q-plate for complex beam shaping," Scientific Reports 6, 1-7 (2016).

[18] Robert C Devlin, Antonio Ambrosio, Noah A Rubin, JP Balthasar Mueller, and Federico Capasso, "Arbitrary spin-to-orbital angular momentum conversion of light," Science 358, 896-901 (2017).

[19] Bruno Piccirillo, Vincenzo D'Ambrosio, Sergei Slussarenko, Lorenzo Marrucci, and Enrico Santamato, "Photon spin-to-orbital angular momentum conversion via an electrically tunable q-plate," Applied Physics Letters 97, 241104 (2010).

[20] Andrew Forbes, "Structured light from lasers," Laser \& Photonics Reviews 13, 1900140 (2019).

[21] Sandile Ngcobo, Igor Litvin, Liesl Burger, and Andrew Forbes, "A digital laser for on-demand laser modes," Nature Communications 4, 1-6 (2013).

[22] Darryl Naidoo, Filippus S Roux, Angela Dudley, Igor Litvin, Bruno Piccirillo, Lorenzo Marrucci, and Andrew Forbes, "Controlled generation of higher-order poincaré sphere beams from a laser," Nature Photonics 10, 327 (2016).

[23] Hend Sroor, Yao-Wei Huang, Bereneice Sephton, Darryl Naidoo, Adam Vallés, Vincent Ginis, Cheng-Wei Qiu, Antonio Ambrosio, Federico Capasso, and Andrew Forbes, "High-purity orbital angular momentum states from a visible metasurface laser," Nature Photonics , 1-6 (2020).

[24] Elhanan Maguid, Ronen Chriki, Michael Yannai, Vladimir Kleiner, Erez Hasman, Asher A Friesem, and Nir Davidson, "Topologically controlled intracavity laser modes based on Pancharatnam-Berry phase," ACS Photonics 5, 1817-1821 (2018).

[25] Ebrahim Karimi, Sebastian A Schulz, Israel De Leon, Hammam Qassim, Jeremy Upham, and Robert W Boyd, "Generating optical orbital angular momentum at visible wavelengths using a plasmonic metasurface," Light: Science \& Applications 3, e167-e167 (2014).

[26] Lixiang Chen, Tianlong Ma, Xiaodong Qiu, Dongkai Zhang, Wuhong Zhang, and Robert W Boyd, "Realization of the Einstein-Podolsky-Rosen paradox using radial position and radial momentum variables," Physical Review Letters 123, 060403 (2019).

[27] E Karimi, RW Boyd, P De La Hoz, H De Guise, J Řeháček, Z Hradil, A Aiello, G Leuchs, and Luis Lorenzo Sánchez-Soto, "Radial quantum number of Laguerre-Gauss modes," Physical Review A 89, 063813 (2014).

[28] Xuemei Gu, Mario Krenn, Manuel Erhard, and Anton Zeilinger, "Gouy phase radial mode sorter for light: Concepts and experiments," Physical Review Letters 120, 
103601 (2018).

[29] Les Allen, Marco W Beijersbergen, RJC Spreeuw, and JP Woerdman, "Orbital angular momentum of light and the transformation of Laguerre-Gaussian laser modes," Physical Review A 45, 8185 (1992).

[30] Marco W Beijersbergen, Les Allen, HELO Van der Veen, and JP Woerdman, "Astigmatic laser mode converters and transfer of orbital angular momentum," Optics Communications 96, 123-132 (1993).

[31] Yijie Shen, Yuan Meng, Xing Fu, and Mali Gong, "Hybrid topological evolution of multi-singularity vortex beams: generalized nature for Helical-Ince-Gaussian and Hermite-Laguerre-Gaussian modes," JOSA A 36, 578587 (2019).

[32] H Laabs and B Ozygus, "Excitation of Hermite-Gaussian modes in end-pumped solid-state lasers via off-axis pumping," Optics \& Laser Technology 28, 213-214 (1996).

[33] YF Chen, TM Huang, CF Kao, CL Wang, and SC Wang, "Generation of Hermite-Gaussian modes in fiber-coupled laser-diode end-pumped lasers," IEEE Journal of Quantum Electronics 33, 1025-1031 (1997).

[34] Yijie Shen, Yuan Meng, Xing Fu, and Mali Gong, "Wavelength-tunable Hermite-Gaussian modes and an orbital-angular-momentum-tunable vortex beam in a dual-off-axis pumped Yb: CALGO laser," Optics Letters 43, 291-294 (2018).

[35] Shu-Chun Chu, Yun-Ting Chen, Ko-Fan Tsai, and Kenju Otsuka, "Generation of high-order HermiteGaussian modes in end-pumped solid-state lasers for square vortex array laser beam generation," Optics Express 20, 7128-7141 (2012).

[36] Weipeng Kong, Atsushi Sugita, and Takunori Taira, "Generation of Hermite-Gaussian modes and vortex arrays based on two-dimensional gain distribution controlled microchip laser," Optics Letters 37, 2661-2663
(2012).

[37] Yijie Shen, Zhensong Wan, Xing Fu, Qiang Liu, and Mali Gong, "Vortex lattices with transverse-mode-locking states switching in a large-aperture off-axis-pumped solid-state laser," JOSA B 35, 2940-2944 (2018).

[38] Yijie Shen, Xilin Yang, Darryl Naidoo, Xing Fu, and Andrew Forbes, "Structured ray-wave vector vortex beams in multiple degrees of freedom from a laser," Optica $\mathbf{7}$, 820-831 (2020).

[39] Zilong Zhang and Changming Zhao, "Spontaneous phase and frequency locking of transverse modes in different orders," Physical Review Applied 13, 024010 (2020).

[40] Zilong Zhang and Changming Zhao, "Intra-cavity generation of linearly distributed and continuously varied optical vortices," IEEE Photonics Technology Letters 32, 196-199 (2020).

[41] Gerard Nienhuis and Steven JM Habraken, "Structure of cavity modes with general astigmatism," Proc. SPIE 6483, Complex Light and Optical Forces 6483, 64830J (2007).

[42] Steven JM Habraken and Gerard Nienhuis, "Orbital angular momentum in twisted and rotating cavity modes," Proc. SPIE 6905, Complex Light and Optical Forces II 6905, 690504 (2008).

[43] Steven JM Habraken and Gerard Nienhuis, "Rotationally induced vortices in optical cavity modes," Journal of Optics A: Pure and Applied Optics 11, 094006 (2009).

[44] Anthony E Siegman, "Lasers university science books," Mill Valley, CA , 617 (1986).

[45] TD Huang and TH Lu, "Large astigmatic laser cavity modes and astigmatic compensation," Applied Physics B 124, 72 (2018).

[46] YF Chen, CC Chang, CY Lee, JC Tung, HC Liang, and KF Huang, "Characterizing the propagation evolution of wave patterns and vortex structures in astigmatic transformations of Hermite-Gaussian beams," Laser Physics 28, 015002 (2017). 